Research Article

\title{
STAP Optimization of Airborne Phased Array Radar in Nonuniform Environment Based on EFA Algorithm
}

\author{
Bin Tang $\mathbb{D},{ }^{1,2}$ Xiaoxia Zheng, ${ }^{1,2}$ Mingxin Liu, ${ }^{1,2}$ and Mengxu Fang ${ }^{1,2}$ \\ ${ }^{1}$ Chengdu Aeronautic Polytechnic, Chengdu 610100, China \\ ${ }^{2}$ College-enterprise Joint Avionics Innovation Base in Sichuan, Chengdu 610100, China \\ Correspondence should be addressed to Bin Tang; liumaocheng@mail.sdufe.edu.cn
}

Received 15 March 2020; Revised 5 August 2020; Accepted 12 August 2020; Published 28 August 2020

Guest Editor: Sanghyuk Lee

Copyright (c) 2020 Bin Tang et al. This is an open access article distributed under the Creative Commons Attribution License, which permits unrestricted use, distribution, and reproduction in any medium, provided the original work is properly cited.

EFA (extended factored approach) algorithm is the main method of space-time adaptive processing technology (STAP) for airborne phased array radar, but it is faced with many problems, such as large number of samples and large amount of calculation. Therefore, this paper uses a method of spatial data dimensionality reduction processing based on cyclic iterative calculation to optimize its STAP. The final experimental results show that, after spatial data dimensionality reduction processing optimization, the STAP performance of EFA algorithm is further expanded in the range of sample number adaptation; especially in the case of small sample number, the optimized STAP performance has been basically close to the ideal compared with other optimization schemes; tap performance also proves that the optimization scheme in this paper has better convergence speed and STAP performance.

\section{Introduction}

In modern air war, airborne radar is the key military technology to win the war, especially the modern early warning aircraft with airborne phased array radar as the main detection means, which is also called the air combat force multiplier of modern war, so the research on airborne phased array radar technology has been endless. The biggest advantage of airborne phased array radar is that its platform can bring it into the high altitude and then scan the airspace through the radio pulse beam in the high altitude, so its detection range and distance are greatly increased. However, in the process of airborne phased array radar scanning the airspace, the transmitted radio pulse beam does not only reflect the objects of interest but also reflect all objects in the airspace. The reflected radio pulse beam either scatters to the surrounding environment or feeds back to the phased array radar antenna array. The radio pulse beam fed back to phased array radar antenna array is processed by signal machine to form scanning information, which includes not only interested target information but also clutter information and interference information. How to eliminate these clutter and interference information has become the key direction to improve the performance of airborne phased array radar. In this study, the main lobe of Doppler filter in space-time adaptive processing technology is easy to be affected by clutter penetration. Two methods, EFA algorithm and spatial decomposition dimension reduction, are used to improve the space-time adaptive processing technology. It is hoped that through this improvement, on the one hand, the computational complexity of space-time adaptive processing technology can be reduced, and on the other hand, the influence of clutter penetration on spacetime adaptive processing technology can be reduced.

In the process of processing clutter information and jamming information, the main technology of airborne phased array radar is space-time adaptive processing technology (STAP), but STAP technology itself has some defects. Especially in the sample size, clutter and noise processing has great limitations. Therefore, based on the analysis of EFA algorithm and STAP technology, this paper proposes an innovative spatial dimension reduction method to optimize the STAP technology performance, so that it can break through the limitation of sample size, further improve the 
ability of STAP to process clutter, and improve the detection ability of airborne phased array radar.

This research includes three parts. The first part is the summary and analysis of STAP technology by domestic and foreign research institutions and scholars. The second part mainly introduces the airborne phased array radar and its clutter model, the related concepts of EFA technology, as well as the detailed airspace dimensionality reduction measures. The third part is to simulate and verify the improved STAP technology of this study and confirm whether the improvement measures of this study can effectively improve the performance of STAP.

\section{Related Work}

Since STAP technology was proposed by Brenna and reed, the related research work has not stopped, and with the emergence of phased array radar technology and frequent military conflicts around the world, more and more attention has been paid and research has been done. In the process of studying the waveform design of fully adaptive radar, scholars Setlur and Rangaswamy used independent iteration to optimize the STAP filter, thus effectively improving the performance of the filter [1]; Jingwei Xu et al. designed a new STAP radar with FDA as the launch array, which can effectively identify the clutter with fuzzy range, and designed a secondary range correlation compensation method to reduce the dependence of the modified radar clutter on the secondary range Aiming at the defects of STAP algorithm, fan team optimized STAP technology by combining principal component analysis and subcpi technology, which greatly improved its convergence speed and proved that this improved method is more practical than traditional technology [2]; Xu et al. designed a new STAP radar with FDA as the launch array, which can effectively identify the clutter with fuzzy range, and designed a secondary range correlation compensation method to reduce the dependence of the modified radar clutter on the secondary range [3]; when McDonalds and Cerutti-Maori study the radar detection technology in the sea clutter environment, they use the actual sea clutter data to simulate the sea targets, and through the two-component clutter model fitting method, they overcome the false alarm problem in the radar detection process and improve the radar detection performance Wang et al. used antenna pulse as the research basis, designed a method that can effectively improve the detection speed of STAP radar system for low target performance, and used min-max iterative algorithm to overcome the problem of antenna pulse selection [4]; $\mathrm{Xu}$ et al. proposed a robust adaptive beamforming method to improve the performance of fda-stap in detecting high-speed targets [5]; Kang et al. in the research of radar system considered that the directivity and resolution of the sensor can be effectively improved when the multiantenna elements are coherent processing the multipulse signal, while the STAP technology can effectively suppress the interference signal [6].

Aiming at the diversity of the transmitting waveform characteristics of airborne MIMO radar, Yidou et al. proposed three kinds of reduced dimension STAP algorithms for MIMO Radar based on the framework structure of reduced dimension STAP, which improved the antijamming performance of MIMO radar [7]. Wang et al. used antenna pulse as the research basis, designed a method that can effectively improve the detection speed of STAP radar system for low target performance, and used min-max iterative algorithm to overcome the problem of antenna pulse selection. Zhou et al. [8] put forward a subspace method based on radar parameters and ellipsoidal wave function to solve the problem that STAP's suppression performance decreases due to the influence of heterogeneous clutter. This method effectively improves STAP's suppression performance in heterogeneous clutter environment [9]; Lin et al. put forward a fast dimension reduction algorithm when studying the clutter suppression of airborne MIMO radar, which can effectively improve the performance of STAP in clutter suppression [10]; when Shi wa et al. studied the problem of target azimuth information hiding caused by the noise interference of STAP radar in the power condensing mode, they proposed a scattering wave interference method, which can effectively improve the performance of STAP radar [11]; Gao et al. put forward a STAP algorithm based on knowledge-assisted sparse recovery to solve the problem that the STAP covariance matrix of airborne radar based on the estimation of clutter covariance matrix of training snapshot which easy to be damaged by the target class signal. This algorithm can effectively identify the clutter [12]; Bruce 1 team proposed a linear transformation process to solve the clutter spectrum dispersion problem of bistatic STAP radar based on the focus matrix, which can effectively improve the clutter suppression ability of STAP radar in nonuniform clutter environment [13].

Wen et al. proposed an enhanced 3D joint domain local STAP method to solve the problem of performance degradation of traditional phased array space-time adaptive processing pseudotarget jamming. This method effectively reduced the radar performance degradation caused by deception jamming by prewhitening filter [14]. When the $s$ team studied the amplitude of time output signal in the detection of weak target by Doppler pre-STAP radar, it was found that linear prediction can improve the performance of Doppler pre-STAP radar more effectively than binomial distribution time weight when determining the weight [15]; $\mathrm{Li}$ aiming at the problem of radar performance degradation caused by space-time adaptive processing space-time steering vector distortion, an anticross coupling space-time adaptive processing method of subarray clutter covariance matrix is proposed. This method can effectively suppress mutual coupling, target component, and target space-time steering vector mismatch in SCM by using target covariance matrix [16]; Chen proposed a two-dimensional amplitude and phase estimation algorithm based on the space-time sliding window of the test unit, which can effectively suppress clutter and interference signals [17]; aiming at the problem that the amount of training data and calculation in $3 \mathrm{D}$ space-time adaptive processing exceeds the actual demand, a method is proposed to convert the plane array data into azimuth and elevation linear array, respectively, so as to form the equivalent cross array before STAP. This method 
can effectively reduce the amount of training data and calculation [18]; the team of Zhi Qi thinks that the STAP algorithm determines the covariance matrix through training sample data, but this process will be destroyed by outliers. Therefore, a robust STAP algorithm is designed based on the joint sparse recovery of clutter spectrum, which can effectively overcome the problem brought by outliers [19]; Jia et al. proposed an online antenna pulse selection method suitable for space-time adaptive processing. The method uses the structural clutter covariance matrix and considers antenna pulse selection and covariance matrix estimation to provide a good objective function for antenna pulse. The experimental results show that this method is feasible [20]. From the comprehensive analysis, there are many researches on STAP performance but few researches on phased array radar, which is one of the important purposes of this paper.

\section{Optimization Measures of Space-Time Adaptive Processing Technology}

3.1. Clutter Model of Airborne Phased Array Radar. The difference between airborne phased array radar and groundbased radar is that compared with the ground, the airborne phased array radar is in motion because of the platform, so the Doppler frequency of ground clutter in the signal received by the airborne phased array radar will have a serious problem of expansion. In some cases, the repetition rate of Doppler broadband pulse of ground clutter will be doubled, and the appearance of STAP technology effectively improves this problem. However, STAP is limited by computation and clutter plus noise covariance matrix, so it has great limitations.

Suppose that the antenna of airborne phased array radar is as shown in Figure 1 and the antenna of radar is arranged in the way of uniform linear array. Then, suppose the number of array elements is $N$, the speed of radar platform is $v_{a}$, the angle between the flight direction and the array direction of radar antenna is $\theta_{p}$, the wavelength and pulse repetition frequency of radar are $\lambda$ and $f_{r}$ respectively, and the number of radar transmitted pulses within the unit coherent processing interval (CPI) is $K$. Then, in a CPI, the clutter data reflected from any clutter scattering point on the ground to the airborne phased array radar system can be described as follows: the airborne phased array radar will meet two conditions during operation, one is the distance between the carrier moving distance in unit CPI and the unit to be detected (cut), and the distance between the two is unchanged; the other is that the clutter echo of each scattering point is statistically independent At the same time, it satisfies the Gauss distribution. Assuming that the distance loop of the point is $l$, the corresponding pitch angle is $\varphi_{l}$ and the azimuth angle of the radar antenna is $\theta_{l}$, then the spacetime sampling data of the radar antenna $n$ received in the $k$ transmission pulse can be expressed by the following formula:

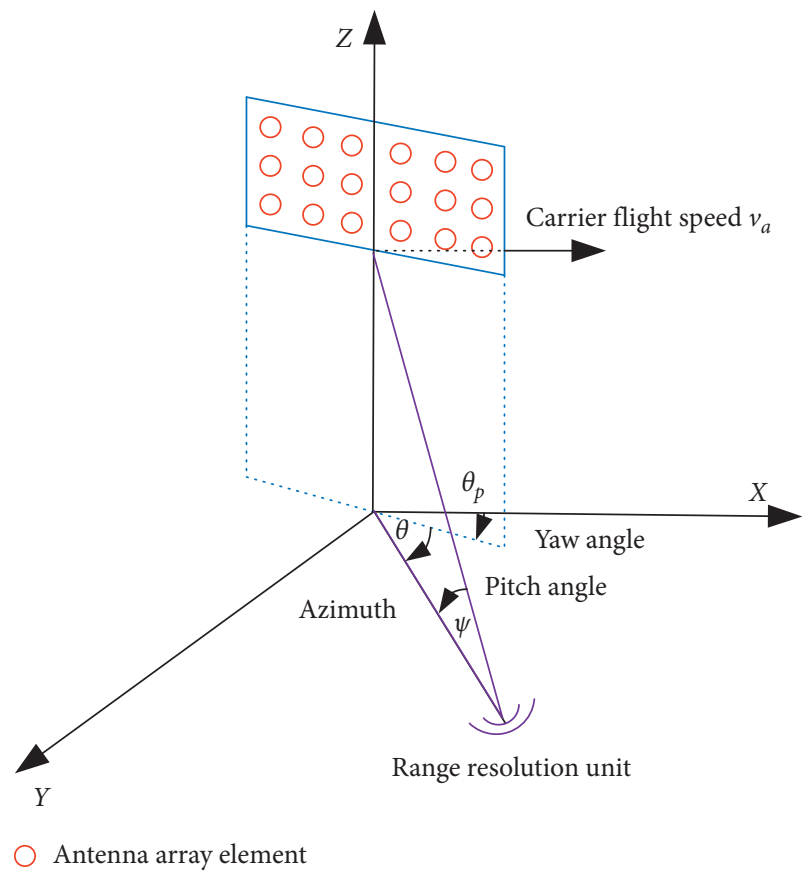

FIGURE 1: Basic structure of airborne phased array radar.

$$
\begin{gathered}
c_{n, k}(\theta, \varphi)=\frac{\rho_{i}}{R_{l}^{2}} F\left(\theta_{i}, \varphi_{l}\right) G\left(\theta_{i}\right) \exp \left\{j 2 \pi \left[(n-1) \omega_{s}\left(\theta_{i}, \varphi_{l}\right)\right.\right. \\
\left.\left.+(k-1) \omega_{t}\left(\theta_{i}, \varphi_{l}\right)\right]\right\}, \quad n=1,2, \ldots, N ; \\
k=1,2, \ldots, K .
\end{gathered}
$$

In the formula, $R_{i}$ represents the distance between radar and scattering point in $l, \rho_{i}$ is the emission coefficient of clutter, $F\left(\theta_{i}, \varphi_{l}\right)$ and $G\left(\theta_{i}\right)$ represent the direction pattern of signal transmission and reception, $\omega_{s}\left(\theta_{i}, \varphi_{l}\right)$ represents the normalized spatial frequency and satisfies $\omega_{s}\left(\theta_{i}, \varphi_{l}\right)=(d / \lambda) \cos \theta_{i} \varphi_{l}$, and $\omega_{t}\left(\theta_{i}, \varphi_{l}\right)$ represents the Doppler frequency and satisfies $\omega_{t}\left(\theta_{i}, \varphi_{l}\right)=\left(2 v / \lambda f_{r}\right) \cos \left(\theta_{i}+\theta_{p}\right) \cos \varphi_{l}$. From these two calculation methods, it can be seen that the normalized airspace frequency is affected by carrier speed and carrier distance, but the carrier is in flight state, so the Doppler frequency is different in different time periods. When $\theta_{p}=0$, the relationship between Doppler frequency and azimuth cosine of clutter on cut is linear; otherwise, it is nonlinear, as shown in Figures 2-4.

Then, for a cut, the clutter echo received by the radar is the sum of all the clutter echoes of scattering points in the cell, and the formula is as follows:

$$
\begin{gathered}
c_{n, k}(\varphi)=\int_{0}^{\pi} \frac{\rho_{i}}{R_{l}^{2}} F\left(\theta_{i}, \varphi_{l}\right) G\left(\theta_{i}\right) \exp \left\{j 2 \pi \left[(n-1) \omega_{s}\left(\theta_{i}, \varphi_{l}\right)\right.\right. \\
\left.\left.+(k-1) \omega_{t}\left(\theta_{i}, \varphi_{l}\right)\right]\right\} \mathrm{d} \theta, \quad n=1,2, \ldots, N ; \\
k=1,2, \ldots, K .
\end{gathered}
$$




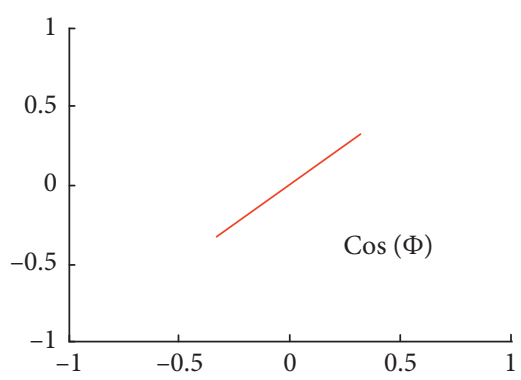

(a)

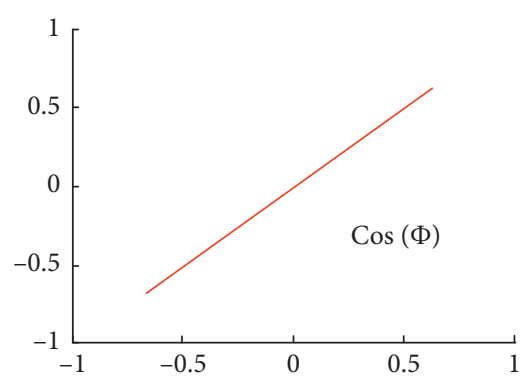

(b)

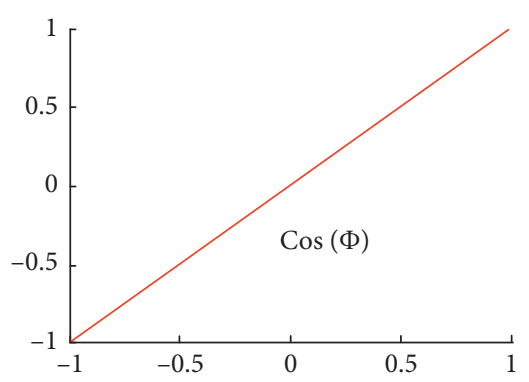

(c)

Figure 2: Clutter trace distribution (front side view array $\theta p=0$ ). (a) Short range. (b) Medium range. (c) Long range.

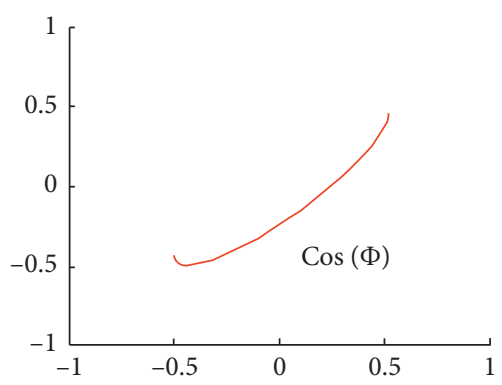

(a)

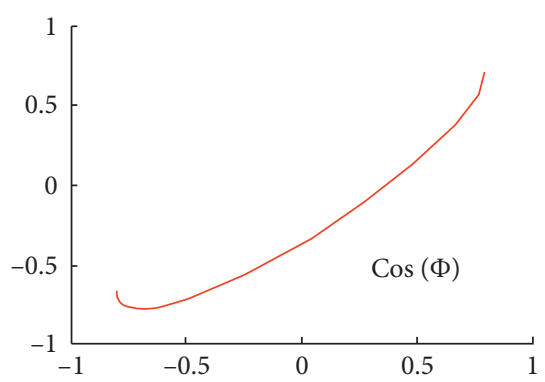

(b)

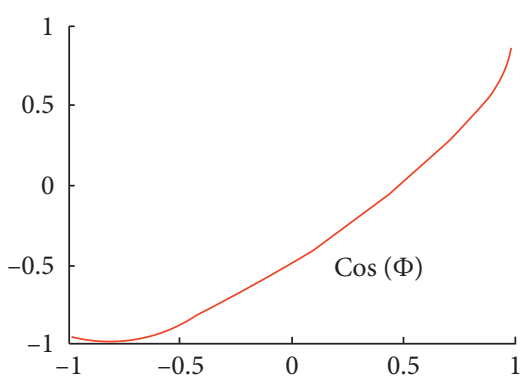

(c)

Figure 3: Clutter trace distribution (squint array $\theta p=0$ ). (a) Short range. (b) Medium range. (c) Long range.

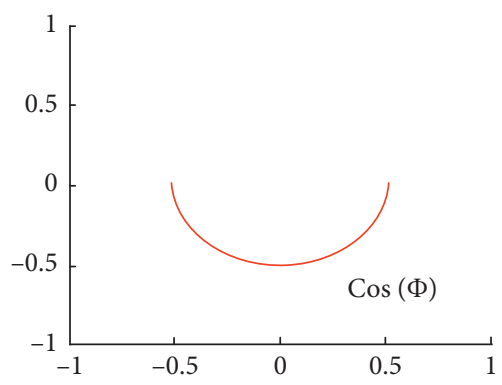

(a)

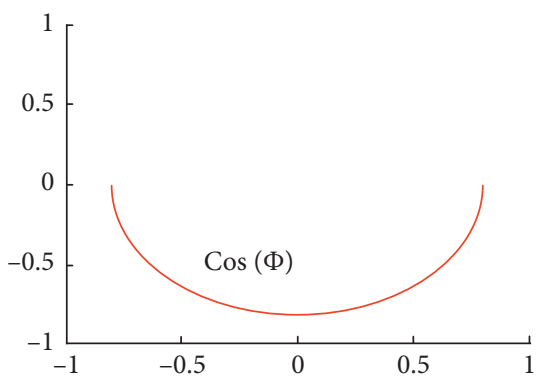

(b)

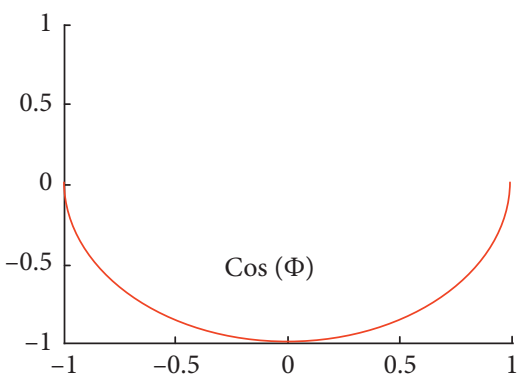

(c)

FIGURE 4: Clutter trace distribution (forward looking array $\theta p=0$ ). (a) Short range. (b) Medium range. (c) Long range.

The result is the sum of the echoes in the range of 0 to $\pi$. The rest of the range cannot be received because the radar backplane is blocked. When the radar receives the sum of the echoes, it will divide the clutter scattering in a cut into $N_{c}$ units. Then, formula (2) can be divided into

$$
\begin{gathered}
c_{n, k}(\varphi)=\sum_{i=1}^{N c} \frac{\rho_{i}}{R_{l}^{2}} F\left(\theta_{i}, \varphi_{l}\right) G\left(\theta_{i}\right) \exp \left\{j 2 \pi \left[(n-1) \omega_{s}\left(\theta_{i}, \varphi_{l}\right)\right.\right. \\
\left.\left.+(k-1) \omega_{t}\left(\theta_{i}, \varphi_{l}\right)\right]\right\}, \quad n=1,2, \ldots, N ; \\
k=1,2, \ldots, K .
\end{gathered}
$$

Then, the clutter received by the radar whose array element number is $N$ and the transmitted pulse number is $K$ can be expressed by the array vector:

$$
\begin{array}{r}
c(l)=\left[c_{1,1}(l), c_{1,2}(l), \ldots, c_{1, K}(l), c_{2,1}(l), \ldots, c_{n, k}(l), \ldots, c_{N, K}(l),\right]^{T}, \\
n=1,2, \ldots, N ; k=1,2, \ldots, K,
\end{array}
$$

where $(\cdot)^{T}$ is the transpose. In view of $l$ and $\varphi_{l}, c_{n, k}(l)$ can be expressed as $c_{n, k}(\varphi)$. In general, there will be noise when the clutter echo is reflected back to the radar, so the echo received in a cut is represented as $x(l)=c(l)+n, n$ as Gaussian white noise signal, and then the covariance matrix between the clutter and noise can be represented as 


$$
R=E\left[x(l) x(l)^{H}\right]
$$

where $(\cdot)^{H}$ represents conjugate transposition and $E[\cdot]$ represents expectation. In general, the covariance matrix is determined by sample estimation. If the clutter distribution characteristics of a cut and its adjacent cut are the same, then its covariance matrix can be estimated by the covariance matrix of adjacent cells, as follows:

$$
\widetilde{R}=\frac{1}{L} \sum_{\rho=1}^{L} x(p) x(p)^{H}
$$

where $L$ represents the number of independent units. However, using this method will lead to the loss of spurious ratio (SCNR) of the output signal. The RMB criterion points out that only when the number of samples exceeds 2 times of matrix dimension can SCNR be less than $3 \mathrm{~dB}$, so the number of matrix samples must be more than 2 times. Finally, the matrix can be transformed into radar clutter spectrum by using space-time Capon spectrum, and the clutter characteristics can be observed by clutter spectrum. The definition of space-time Capon spectrum is

$$
p(\varphi, \theta)=\frac{1}{s^{H}(\varphi, \theta) R^{-1} s(\varphi, \theta)},
$$

where $s(\varphi, \theta)$ represents two-dimensional search oriented vector and $(\cdot)^{-1}$ represents matrix inversion.

The above is the process of building the clutter model of airborne phased array radar.

3.2. Space-Time Adaptive Processing Technology and Extended Factored Approach. In general, the signals reflected back to the airborne phased array radar can be represented by the following two assumptions:

$$
\begin{aligned}
& H_{0}: s(l)=c(l)+n, \\
& H_{1}: x(l)=c(l)+n+\beta s,
\end{aligned}
$$

where $\beta$ is the normalized Doppler frequency of the target and other symbols are the same as the above, where $s=s_{t} \otimes s_{s}, s_{t}, s_{s}$ represents the time domain and space domain guidance vectors of the target, respectively, which are calculated by the following formula:

$$
\begin{aligned}
& s_{t}=\left[1, \exp j 2 \pi \omega_{t}, \ldots, \exp j 2 \pi(K-1) \omega_{t}\right], \\
& s_{s}=\left[1, \exp j 2 \pi \omega_{s}, \ldots, \exp j 2 \pi(N-1) \omega_{s}\right] .
\end{aligned}
$$

In the reflected signal, clutter, noise, and target signal are all independent of each other. The radar samples the spatial and temporal data by the combination of antenna array and transmitting pulse, and STAP filters the data. Suppose $w$ is the weight vector of STAP processor, then the output result is

$$
z=w^{H} x(l)
$$

The final purpose of STAP is to identify the target signal, so the selection of the optimal weight vector of STAP processor can be solved by the following formula:

$$
\begin{cases}\min & E\left|w^{H} x(l)\right|^{2}=w^{H} R w \\ \text { s.t. } & w^{H} s=1 .\end{cases}
$$

The main purpose of this formula is to suppress clutter when the signal energy is constant, so as to minimize the output capacity of clutter and noise. The solution of $w$ is obtained by Lagrange multiplier method, as follows:

$$
w=\gamma R^{-1} s,
$$

where $\gamma$ represents the normalized constant. According to the above formula, STAP processor is a generalized Wiener filter. The improvement factor (IF) measures the key indicators of STAP processor, which can be defined as

$$
\mathrm{IF}=\frac{\mathrm{SCNR}_{\text {out }}}{\mathrm{SCNR}_{\text {in }}},
$$

where $\mathrm{SCNR}_{\text {out }}$ and $\mathrm{SCNR}_{\text {in }}$ represent the signal to noise ratio of output and input, respectively, where

$$
\operatorname{SCNR}_{\text {out }}=s^{H} R^{-1} s \text {. }
$$

If the input power of clutter and noise is set to $\sigma_{c}$ and $\sigma_{w}$, respectively, then

$$
\operatorname{SCNR}_{\text {out }}=\frac{1}{\sigma_{c}+\sigma_{w}}=\frac{1}{(\mathrm{CNR}+1) \sigma_{w}},
$$

where CNR in represents the noise ratio, and then IF of the optimal STAP is expressed as

$$
\mathrm{IF}=(\mathrm{CNR}+1) \sigma_{w} s^{H} R^{-1} s .
$$

The above is the whole process of STAP, but the airborne phased array radar directly uses STAP technology to process the clutter signal, which requires a lot of hardware consumption, computation, and clutter covariance matrix. Therefore, in the practical application of STAP dimension reduction processing, other methods should be combined to reduce the use cost of STAP. This paper first introduces the EFA algorithm used in this paper.

In the process of STAP application, the Doppler filter with high out band attenuation can effectively suppress clutter, but the main lobe of Doppler filter will have the problem of clutter infiltration, so we need to take adaptive processing method to solve this problem. The main purpose of EFA algorithm is to use the data of Doppler channel and its adjacent channels to complete the adaptive processing. The basic principle of its structure is shown in Figure 5. The adjacent channel is the auxiliary channel, and the rest is the main channel, so as to improve the robustness of the time domain.

Assuming $\quad \tilde{y}_{k}(l)=\left[\tilde{x}_{K-1}^{T}(l), \tilde{x}_{k}^{T}(l), \tilde{x}_{k+1}^{T}(l)\right]^{T} \quad$ and $\widetilde{S}_{k}=\left[\widetilde{s}_{k-1}^{T}, \tilde{s}_{k}^{T}, \tilde{s}_{k+1}^{T}(l)\right]^{T}$, the constraint equations of three Doppler channels in signal processing can be expressed as follows: 


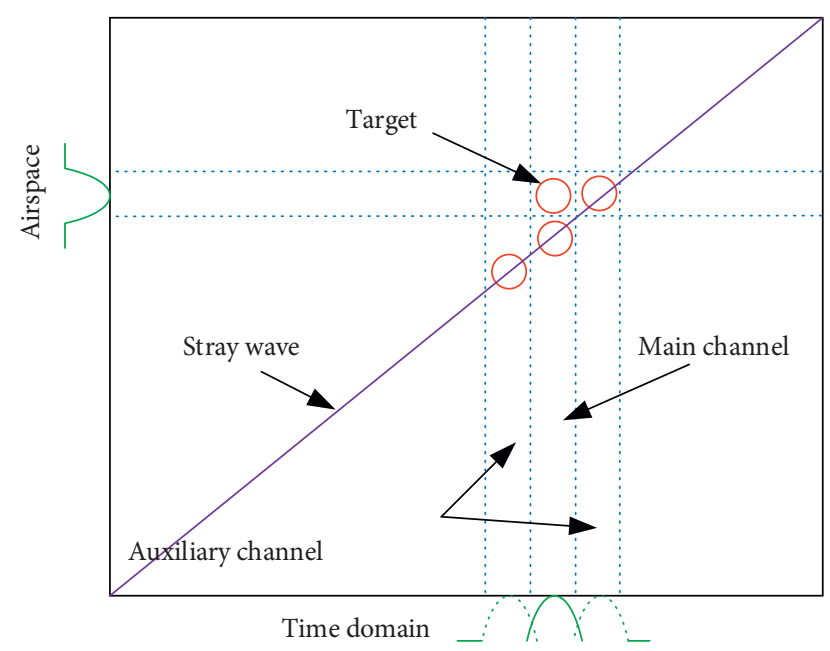

Figure 5: EFA fundamentals.

$$
\begin{cases}\min & E\left[\left|\widetilde{w}_{\mathrm{EFA}}^{H} \tilde{y}_{k}(l)\right|\right]^{2} \\ \text { s.t. } & \widetilde{w}_{\mathrm{EFA}}^{H} \widetilde{S}_{K}=1 .\end{cases}
$$

The optimal solution of the formula is expressed as

$$
\widetilde{w}_{\mathrm{EFA}}^{H}=\frac{R_{\tilde{y}_{k}}^{-1} \widetilde{S}_{k}}{\widetilde{S}_{k}^{H} R_{\widetilde{y}_{k}}^{H} \widetilde{S}_{k}} .
$$

Corresponding $R_{\widetilde{y}_{k}}=E\left[\widetilde{y}_{k}(l) \widetilde{y}_{k}^{H}(l)\right]$, according to the RMB criterion, the number of independent training samples required by EFA is well controlled, and the amount of calculation is further reduced.

\subsection{Optimization of Space-Time Adaptive Processing} Technology. The main process of post-Doppler adaptive processing (FA) is to suppress the main clutter by using time-domain Doppler and then carry out spatial adaptive processing. Therefore, the adaptive processing can be carried out in multiple Doppler channels at the same time. Here, it is assumed that the filter coefficient vector of Doppler channel $k, k=1,2,3, \ldots, K$, is $f_{k}=\left[w_{k}^{0}, w_{k}^{1}, w_{k}^{2}, \ldots, w_{k}^{K-1}\right]^{T}$, where $w_{k}^{l}=\exp \left(j 2 \pi \bar{f}_{k} l\right), \overline{f k}=(k-1 / K) f_{r}$ in the formula and $l=0,1,2, \ldots, K-1$. In the combination of multiple Doppler filters, the center frequency of each filter is different, but the amplitude characteristics are the same. Then, the response function of amplitude is a periodic function with a period of $f_{r}$, as shown in Figure 6. Figure 6 is the amplitude response curve of a Doppler filter with a center frequency of $0.5 f_{r}$. Between the first pair of zeros, the main response width of the filter is $2 f_{r} / N$, while the width of the corresponding half power is about $0.9 f_{r} / N$, while the height ratio of the main lobe to the side lobe of the filter is $13.2 \mathrm{db}$. In this process, the high sidelobe is easy to cause clutter leakage and ultimately affect the performance of Doppler filter adaptive processing, so it must be suppressed.

After Doppler channel filtering, the data of clutter and noise in the signal can be expressed by the following formula:

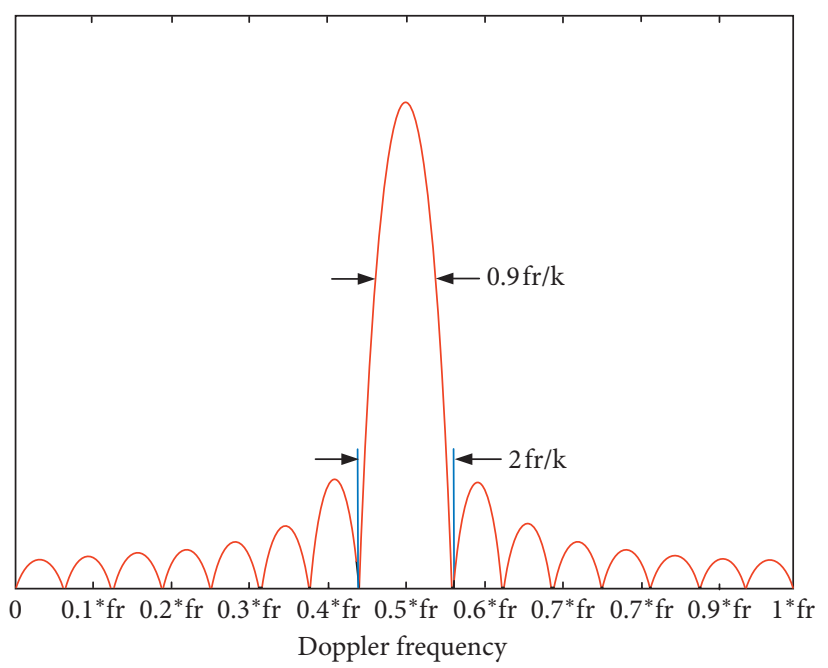

Figure 6: Amplitude response of a single number of Doppler filters.

$$
\tilde{x}_{k} l=f_{k}^{K} \otimes I_{N} x(l)
$$

The formula $N \times N$ represents unit matrix. Suppose that the target's guiding vector $s=s_{t} \otimes s_{s}$ and $s_{t}, s_{s}$ represent the target's guiding vector in time domain and space domain, respectively. See formula (9) for the calculation method of $s_{t}$, $s_{s}$, and the data of the target's guiding vector after $k$ filtering will become

$$
\tilde{x}_{k}=\left(f_{k}^{K} \otimes I_{N}\right) s .
$$

According to the RMB criterion, FA processing is used to keep the energy of $k$ target signal unchanged, and then the output ability of clutter is reduced to achieve the purpose of clutter suppression:

$$
\begin{cases}\min & E\left[\widetilde{w}_{\mathrm{FA}}^{H} \widetilde{x}_{k}(l)\right]^{2} \\ \text { s.t. } & \widetilde{w}_{\mathrm{EFA}}^{H} \widetilde{S}_{K}=1 .\end{cases}
$$

The optimal solution can also be obtained by using Lagrange formula:

$$
\widetilde{w}_{\mathrm{FA}}^{H}=\frac{R_{\tilde{x}_{k}}^{-1} \widetilde{S}_{k}}{\widetilde{S}_{k}^{H} R_{x_{k}}^{-1} \widetilde{S}_{k}},
$$

where $R_{\widetilde{x}_{k}}=E\left[\widetilde{x}_{k}(l) \tilde{x}_{k}^{H}(l)\right]$ is the covariance matrix of the Doppler channel $k$. For EFA, according to formulas (17) and (18), the optimal solution can also be obtained, and there is $R_{\widetilde{y}_{k}}=E\left[\widetilde{y}_{k}(l) \widetilde{y}_{k}^{H}(l)\right]$. However, in the actual processing, the clutter and noise data of the unit distance unit adjacent to the target unit of the covariance matrix can be obtained. Then, according to formula (6), $R_{\widetilde{x}_{k}}$ and $R_{\tilde{y}_{k}}$ can be obtained:

$$
\begin{aligned}
& \widehat{R}_{\widetilde{x}_{k}}=\frac{1}{L} \sum_{l=1}^{L} \tilde{x}(l) \tilde{x}_{k}^{H}(l), \\
& \widehat{R}_{\widetilde{y}_{k}}=\frac{1}{L} \sum_{l=1}^{L} \tilde{y}(l) \widetilde{y}_{k}^{H}(l),
\end{aligned}
$$


where $L$ has the same meaning as in formula (6). Theoretically, when the number of training samples of Doppler filter bank $L \longrightarrow \infty$, there should be $R_{\bar{y} k}=\widehat{R}_{\bar{y}_{k}}$ and $R_{\bar{x} k}=\widehat{R}_{\bar{x}_{k}}$, However, from a practical point of view, the number of samples cannot be infinite and some studies have proved that, when the number of samples is more than twice the number of covariance matrix, the output loss of signal to noise ratio will not be greater than $3 \mathrm{~dB}$. Although the number of samples and the amount of calculation have decreased, but when the number of radar antenna elements increases, the number of samples will increase, eventually leading to the reduction of clutter suppression performance. Therefore, further dimension reduction is needed.

The clutter data processed by Doppler channel $k$ can be expressed by the following formula when dimension reduction is carried out from the perspective of spatial data:

$$
\widetilde{z}_{k}(l)=\sum_{i=1}^{N c} \alpha_{i} c_{s, i},
$$

where $\alpha_{i}=\left(\sigma_{i}\left(\theta_{l}, \varphi_{i}\right) / R_{l}^{2}\right) F \theta_{l}, \varphi_{i} G \theta_{l} f_{k}^{H} c_{t, i}$ is a constant. It can be seen from the above formula that the filtered data become the product of the spatial guiding vector of the clutter and a constant, which belongs to the column vector, and the size of the column vector is $N \times 1$, while $c_{s, i}$ can continue to be represented by the Kronecker product of the short vector, as follows:

$$
\begin{aligned}
c_{s, i}= & {\left[1, \ldots, \exp \left(2 j \pi\left(N_{1}-1\right) N_{2} \omega_{s, i}\right)\right] T } \\
& \otimes\left[1, \ldots, \exp j 2 \pi\left(N_{2}-1\right) \omega_{s, i}\right]^{T}, \\
a_{i}= & {\left[1, \exp \left(2 j \pi N_{2} \omega_{s, i}\right), \ldots, \exp \left(2 j \pi\left(N_{1}-1\right) N_{2} \omega_{s, i}\right)\right]^{T}, } \\
b_{i}= & {\left[1, \exp \left(j 2 \pi \omega_{s, i}\right), \ldots, \exp \left(j 2 \pi\left(N_{2}-1\right) \omega_{s, i}\right)\right] T . }
\end{aligned}
$$

The above two formulas are the column vectors of $N_{1} \times 1, N_{2} \times 1$, and $N=N_{1} N_{2}$; then, the formula (24) can be expressed as

$$
\widetilde{z}_{k}(l)=\sum_{i=1}^{N c} \alpha_{i} c_{s, i}=\sum_{i=1}^{N c} \alpha_{i} a_{i} \otimes b_{i} .
$$

Then, formula (17) is in the form of

$$
\widetilde{w}_{\mathrm{FA}}=u \otimes v,
$$

where $u=\left[u_{1}, u_{2}, \ldots, u_{N 1}\right]^{T} v=\left[v_{1}, v_{2}, \ldots, v_{N 2}\right]^{T}$; substituting formula (27) into formula (17), we can get

$$
\begin{cases}\min & E\left[\left|\widetilde{w}_{\mathrm{FA}}^{H} \widetilde{x}_{k}(l)\right|\right]^{2}=E\left[\left|(u \otimes v)^{H} \widetilde{x}_{k}(l)\right|^{2}\right] \\ \text { s.t. } & \widetilde{w}_{\mathrm{FA}}^{H} \widetilde{s}_{k}=(u \otimes v)^{H} \widetilde{s}_{k}=1 .\end{cases}
$$

Formula (28) becomes a biquadratic cost function of vector $u$ and vector $v$. Then, according to the theory of cycle minimization, $u$, or $v$, get the initial value randomly and then substitute it into formula (28) to solve until the final error value is less than the threshold value. That is, fix the vector $u$ first, give it a specified initial value, write it down as $u(0)$, and then substitute it into formula (28), you can get

$$
\begin{cases}\min & E\left[\left|(u(0) \otimes v)^{H} \widetilde{x}_{k}(l)\right|^{2}\right]=E\left[\left|v^{H}\left(u(0) \otimes I_{N 2}\right) \widetilde{x}_{k}(l)\right|^{2}\right] \\ \text { s.t. } & (u(0) \otimes v)^{H} \widetilde{\mathcal{s}}=v^{H}\left(u(0) \otimes I_{N 2}\right)^{H} \widetilde{\mathcal{s}}=1,\end{cases}
$$

where $\mathrm{IN}_{2}$ is the unit matrix of $N_{2} \times N_{2}$, and if it is named $R_{v}=\left(u(0) \otimes I_{N 2}\right)^{H} R_{\widehat{x}_{k}}\left(u(0) \otimes I_{N 2}\right), \quad s_{v}=\left(u(0) \otimes I_{N 2}\right)^{H} \widetilde{\mathcal{s}}$, then we can get

$$
v(1)=\frac{R_{v}^{-1} s_{v}}{s_{v}^{H} R_{v}^{-1} s_{v}}
$$

Then, the obtained $v(1)$ value is substituted into formula (28) to continue the iterative calculation, and

$$
\begin{cases}\min & E\left[\left|(u \otimes v(1))^{H} \widetilde{x}_{k}(l)\right|^{2}\right]=E\left[\left|u^{H}\left(I_{N 1} \otimes v(1)\right)^{H} \widetilde{x}_{k}(l)\right|^{2}\right] \\ \text { s.t. } & (u \otimes v(1))^{H} \widetilde{s}=u^{H}\left(I_{N 1} \otimes v(1)\right)^{H} \widetilde{s}=1,\end{cases}
$$

where $\mathrm{IN}_{1}$ is $N_{1} \times N_{1}$ 's unit matrix. Similarly, $R_{u}=\left(I_{N 1} \otimes v(1)\right)^{H} R_{\widehat{x}_{k}}\left(I_{N 1} \otimes v(1)\right), s_{u}=\left(v(1) \otimes I_{N 1}\right)^{H} \widetilde{s}$, we can get

$$
u(1)=\frac{R_{u}^{-1} s_{u}}{s_{u}^{H} R_{u}^{-1} s_{u}} .
$$

Cycle the steps of the above iteration until the $k$ step is reached. $\|v k-v k-1\| /\|v k\|<\delta, \delta$ is the error threshold and $\|\cdot\|$ is the 2 norm of the vector. In the process of iteration, the Doppler covariance matrix of phased array meets $R_{v} \in C^{N_{2} \times N_{2}}$ and $R_{u} \in C^{N_{1} \times N_{1}}$, respectively. Then, by decomposing the spatial data, the training samples of covariance matrix only need to be larger than $2 \max \left(N_{1}, N_{2}\right)$, and the closer $N_{1}, N_{2}$ value is, the smaller the sample size is.

\section{Result Analysis}

4.1. Convergence and Computational Analysis. In this paper, the convergence and computation of the optimized STAP are verified and analyzed. First, the life cost function is

$$
J(u, v)=E\left[\left|(u \otimes v)^{H} \widetilde{x}_{k}(l)\right|^{2}\right]+\mu\left[(u \otimes v)^{H} \widetilde{s}_{k}-1\right] .
$$

According to the formula, it can be seen that the cost function is continuous and differentiable. Substituting $u(0), \ldots, u(k), v(1), \ldots, v(k)$ is calculated in the previous section with the cost function $J(u, v)$; the following results can be obtained:

$$
\begin{aligned}
J(u(0), v(1)) & \geq J(u(1), v(1)) \geq \cdots \geq J(u(k-1), v(k-1)) \\
& \geq J(u(k-1), v(k)) \geq J(u(k), v(k)) .
\end{aligned}
$$

In the above results, it is obvious that the results of each cycle iteration satisfy the monotonic decrease. A $\|v\|=1$, you can get the following results: 


$$
J(u(t), v(t))=\bar{J}(w)=w^{H} R_{\widetilde{x} k} w \geq \lambda_{\min }\|w\|,
$$

where $\lambda_{\min }$ represents the minimum eigenvalue of the covariance matrix $R_{\widetilde{x}_{k}}$ of STAP. This result shows that there is a constant of $0<c<+\infty$, let $\lambda_{\text {min }}\|w\| \leq \bar{J}(w)=w^{H} R_{\tilde{x}_{k}} w<c$ hold, this result shows that the cost function is bounded. According to the above analysis, we can know that $J(u(t), v(t))$ is an Lyapunov function, and it further shows that the iterative algorithm in the previous section has the characteristics of progressive convergence.

After it is proved that the iterative cycle adopted in this paper for STAP optimization has convergence, the sample size calculated by several algorithms is shown in Table 1, and the last sdd-fa and sdd-efa are the optimized algorithms in this paper. It can be seen from the table that the number of full-dimensional space-time adaptive samples is 2048 and the calculation amount reaches 109 , while the number of samples of FA algorithm and EFA algorithm is 128 and 384, respectively. In this paper, the number of samples of FA algorithm and EFA algorithm after spatial decomposition and dimension reduction optimization is 16 and 32 , respectively, and the calculation amount is also within 103 . The results show that the number of samples and the amount of computation of FA algorithm and EFA algorithm are greatly optimized after the optimization processing of spatial decomposition and dimensionality reduction, which shows that the STAP performance of FA algorithm and EFA algorithm is greatly improved.

4.2. Performance Simulation of Improvement Factor. In this paper, the optimized STAP performance is simulated. In general, the performance of STAP is measured by improvement factor. Therefore, it is assumed that the data of airborne phased array radar and other relevant data involved in the experiment are shown in Table 2 , in which the array of experimental airborne phased array radar is $64 \times 64$, the number of pulses in the unit coherent processing compartment $K=16$, the pulse repetition frequency $f_{r}=2000 \mathrm{~Hz}$, the wave length of radar emission $\lambda=0.2 \mathrm{~m}$, the flight height and speed of radar carrier are $9000 \mathrm{~m}$ and $100 \mathrm{M} / \mathrm{s}$, respectively, the spurious ratio is $60 \mathrm{~dB}$, and the clutter relative broadband is set as 0.02 .

Figure 7 shows the results of improvement factor performance at 500 samples. The purple dotted line opt in the figure represents the optimal STAP, and this curve is mainly used as a reference. The rest are several algorithms involved in the experiment. It can be seen from the figure that, as the normalized Doppler frequency increases from small to large, the improvement factors of all algorithms appear as a decline rise process. When the normalized Doppler frequency value is 0 , the improvement factors of all algorithms are minimized. But compared with the opt curve, we can see that the EFA algorithm and the EFA algorithm (sdd-efa), which are optimized by spatial data dimensionality reduction, have the best improvement factor. The improvement factor data of FA algorithm and EFA algorithm (sdd-fa) are basically the same, but they are far from the ideal data.
TABLe 1: Performance comparison between different algorithms.

\begin{tabular}{lcc}
\hline Algorithm & \multicolumn{1}{c}{ Index } \\
& Amount of computation & Sample size \\
\hline Full-dimension STAP & $O\left(10^{9}\right)$ & 2048 \\
FA & $O\left(10^{5}\right)$ & 128 \\
EFA & $O\left(10^{6}\right)$ & 384 \\
SDD-FA & $O\left(10^{2}\right)$ & 16 \\
SDD-EFA & $O\left(10^{3}\right)$ & 32 \\
\hline
\end{tabular}

TABLE 2: Experimental phased array radar and other relevant data.

\begin{tabular}{lcc}
\hline Name & Symbol & Numerical value \\
\hline Airborne phased array radar array & - & $64 \times 64$ \\
Number of pulses & $K$ & 16 \\
Pulse repetition rate & $f r$ & $2000 \mathrm{~Hz}$ \\
Radar wavelength & $\lambda$ & $0.2 \mathrm{~m}$ \\
Flight altitude of carrier & $h_{a}$ & $9000 \mathrm{~m}$ \\
Carrier flight speed & $v_{a}$ & $100 \mathrm{~m} / \mathrm{s}$ \\
Clutter relative broadband & $B_{r}$ & 0.02 \\
Heterozygosity ratio & $\mathrm{CNR}$ & $60 \mathrm{~dB}$ \\
\hline
\end{tabular}

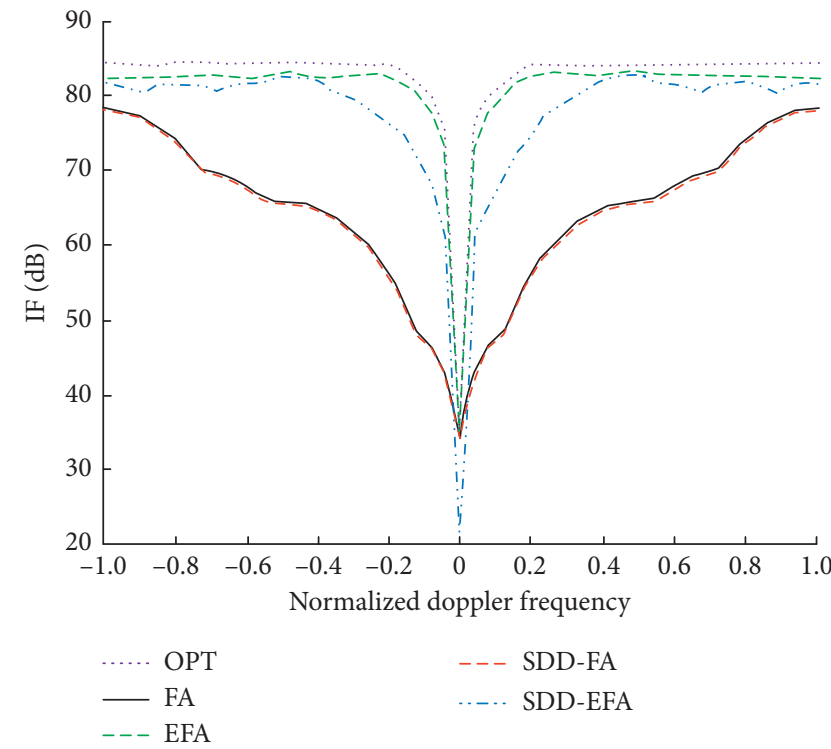

FIgURE 7: Performance comparison of improvement factors under 500 samples.

Figure 8 shows the statistical results of the improvement factor curve of the four algorithms and the ideal improvement factor curve when the number of samples is 50. It can be seen from the figure that, in the case of a small number of samples, the improvement factor curves of the four algorithms are greatly different. The FA algorithm is the farthest from the ideal curve, and the improvement factor curve of the sdd-efa algorithm is the closest to the ideal curve, which shows that the performance of the algorithm is the closest to the ideal STAP performance. Sddfa algorithm and EFA algorithm are relatively similar. The performance of STAP in different normalized Doppler frequencies is staggered. The STAP performance of EFA 


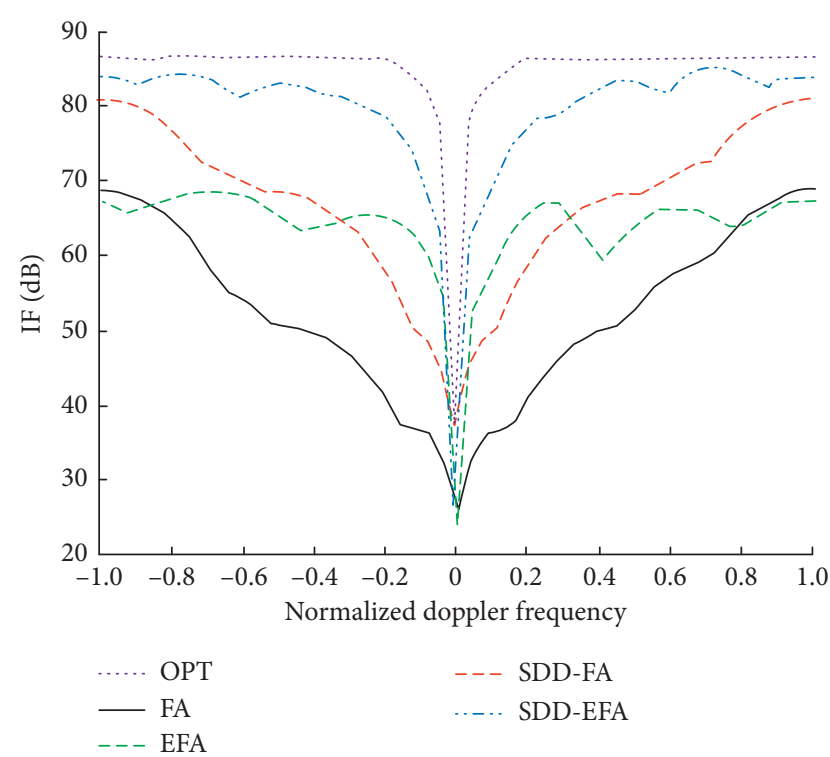

FIGURE 8: Comparison of improvement factor performance under a number of samples.

algorithm in Doppler frequency $-0.2 \sim 0.2$ is better. The rest of the frequency curves are more prominent in sdd-fa algorithm, but the STAP performance of EFA in different frequencies is more stable.

Figure 9 shows the performance comparison of EFA algorithm and sdd-efa algorithm under different training samples. As can be seen from the figure, with the increase of the number of training samples, the improvement factors of the sdd-efa algorithm show an upward stable trend. When the number of samples is 50 , the curve area is stable, which shows that the STAP performance of the sdd-efa algorithm tends to be stable when the number of samples exceeds 50, while the EFA algorithm starts to appear when the number of samples is 200 and tends to be stable when the number of samples is 350 . The comparison shows that the STAP sample of sdd-efa algorithm has a wider range of applicability.

Figure 10 shows the relationship between the sdd-efa algorithm and the number of iterations. It can be seen from the figure that with the increase of the number of iterations, the improvement factor of the sdd-efa algorithm increases gradually and reaches a stable stage after nine iterations. The result shows that the performance of the SATP tends to be stable when the number of iterations exceeds nine.

4.3. Performance Analysis of Clutter Removal. The experimental results of STAP performance in the sidelobe region of phased array radar are shown in Figures 11-14. Figure 11 shows the statistical results of STAP performance of four algorithms in the Sidelobe region. It can be seen from the figure that, with the gradual increase of the normalized Doppler frequency, the STAP performance of the four algorithms also experienced a downward to upward trend, and the improvement factors of all algorithms reached the lowest near 0.6. But in the whole stage, the biggest improvement

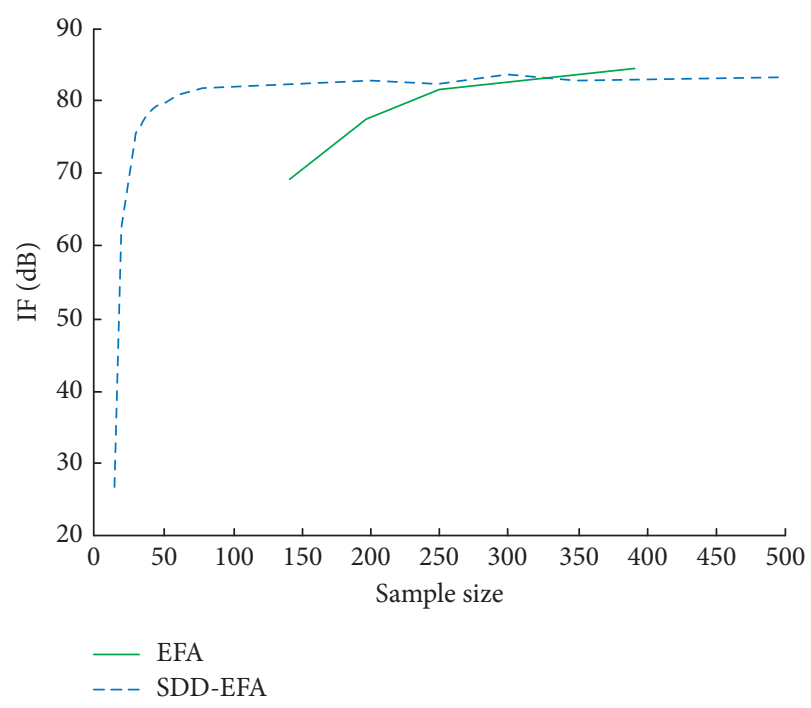

FIgURE 9: Performance comparison of EFA algorithm and sdd-efa algorithm under different training samples.

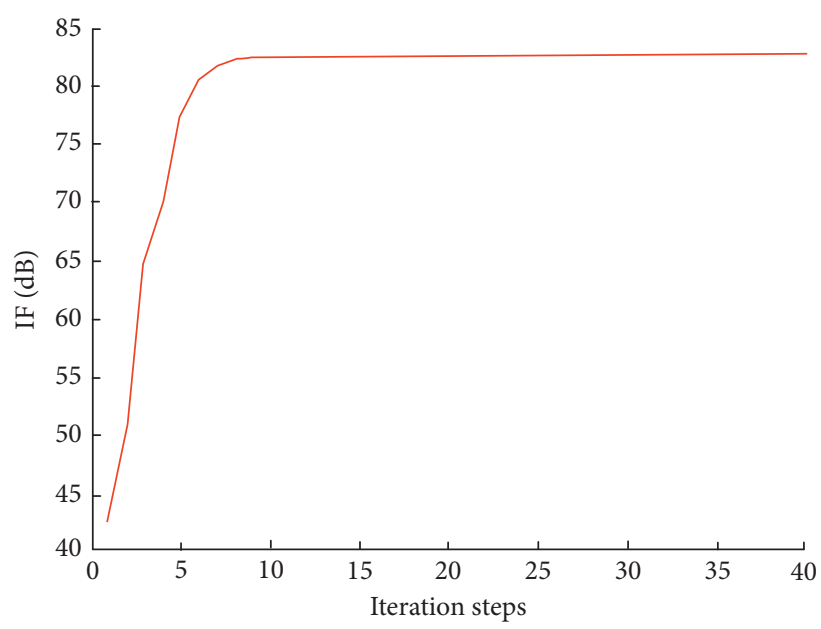

FIGURE 10: Relationship between the sdd-efa algorithm and the number of iterations.

factor is still the sdd-efa algorithm, followed by the sdd-fa algorithm. The two are close in most of the normalized Doppler frequency range, but between 0.3 and 1.0, the performance of sdd-efa algorithm is better. FA fluctuates with the frequency of normalized Doppler, and the performance of EFA is very unstable.

Figure 12 shows the change trend of improvement factors of EFA algorithm and sdd-efa algorithm in sidelobe clutter area under different training samples. It can be seen from the figure that the trend of STAP performance of the two algorithms in the side lobe clutter area with the number of samples is basically similar to the result shown in Figure 9, that is, the STAP performance of the sdd-efa algorithm in the side lobe clutter area is more extensive in the range of the number of training samples, and the advantages are more obvious. 


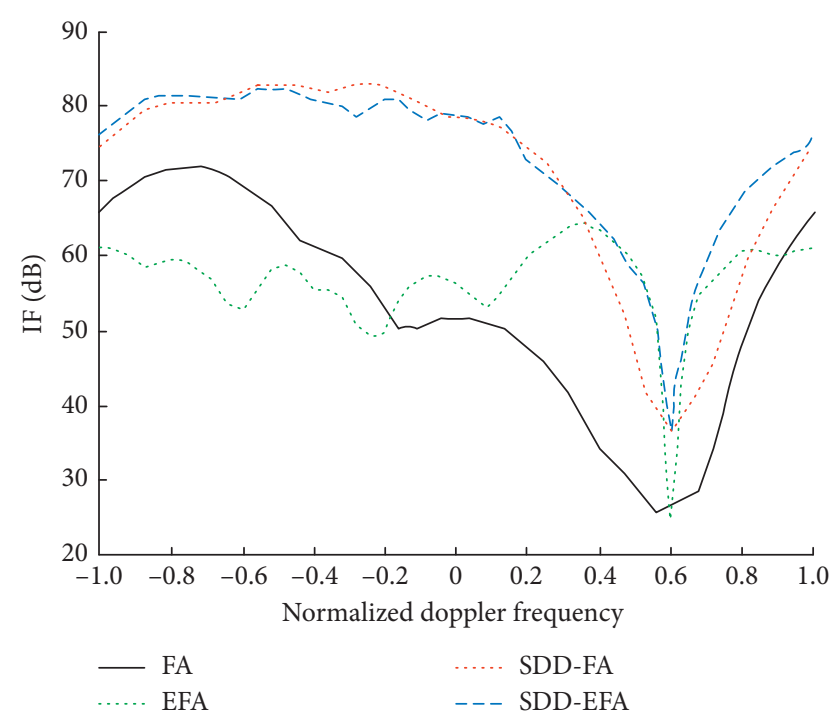

FIgURE 11: Performance comparison of STAP improvement factors in side lobe area.

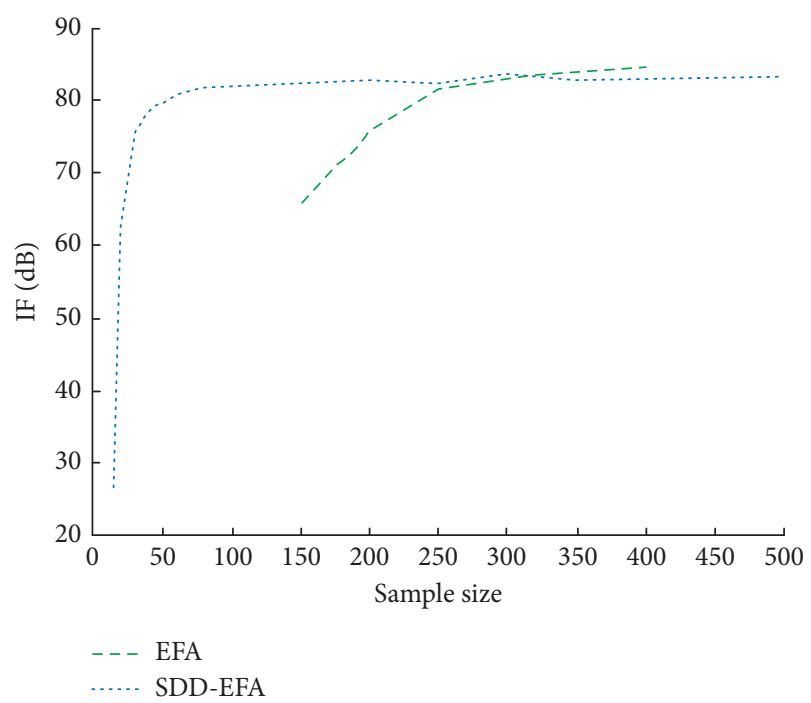

FIGURE 12: Comparison of sidelobe clutter STAP convergence performance.

In order to verify the advantages and disadvantages of this optimization scheme compared with other optimization schemes, we choose to compare it with pc-efa algorithm. Figure 13 shows the improvement factor comparison between pc-efa algorithm and sdd-efa algorithm in 50 samples. It can be seen from the figure that the improvement factor values of the two optimization schemes are relatively close, but the improvement factor values of the optimization scheme in this paper are larger than those of the pc-efa algorithm, which is basically kept at about $80 \mathrm{~dB}$, while the pc-efa algorithm is kept at about $75 \mathrm{db}$.

Figure 14 shows the improvement factor performance comparison of the two optimization schemes at different sample numbers. It can be seen from the figure that, in the initial stage, the improvement factor performance of pc-efa

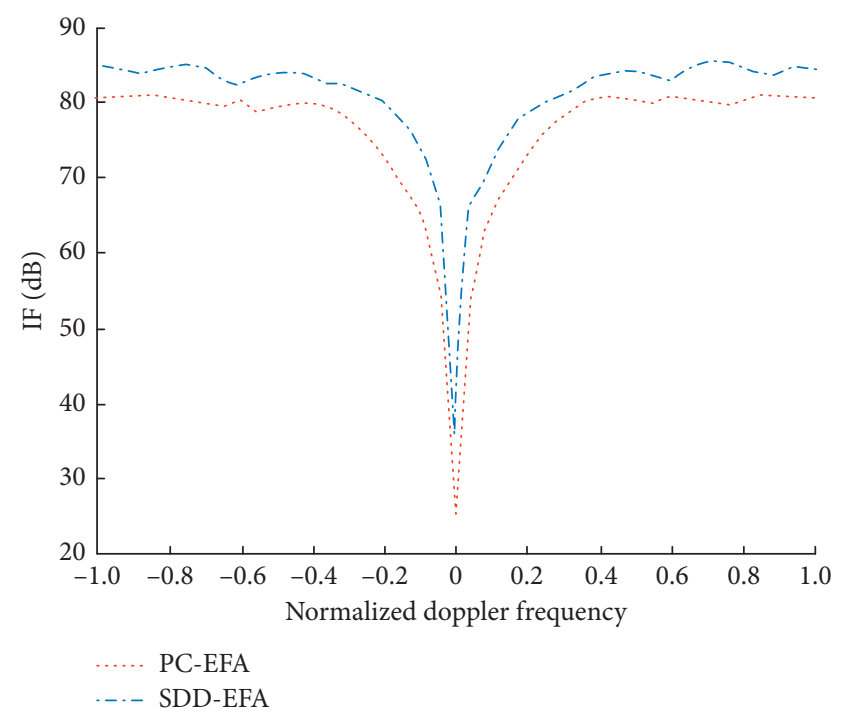

FIGURE 13: Comparison of morning STAP performance of two different optimization schemes with 50 samples.

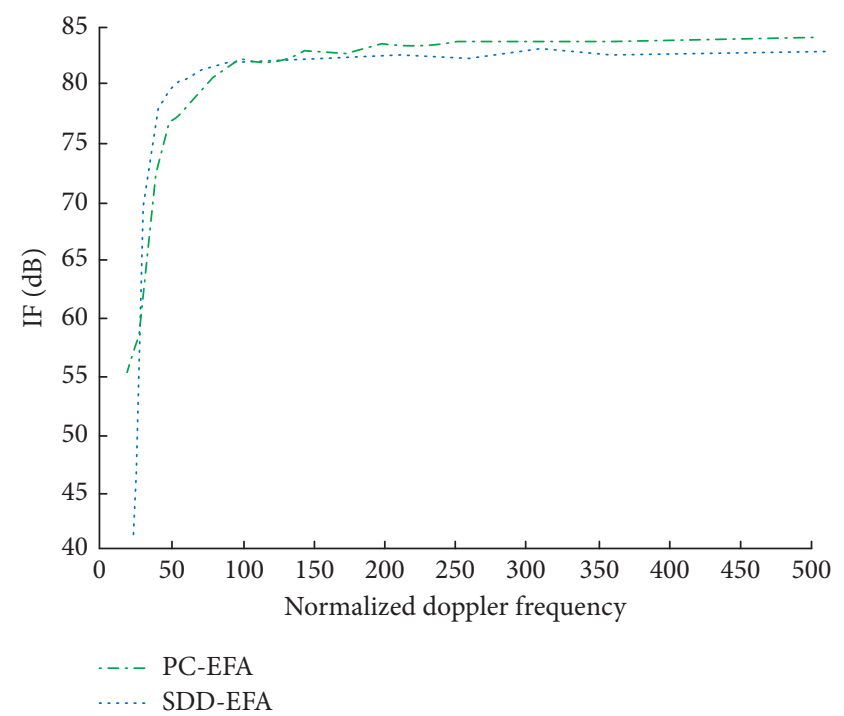

FIGURE 14: STAP performance comparison of two different optimization schemes under different sample numbers.

algorithm is better than that of this algorithm, but with the increase of the number of samples, the improvement factor performance of this optimization scheme gradually exceeds that of pc-efa algorithm, and the two are basically close when the number of samples is 100 . In terms of the convergence speed, the convergence speed of this optimization scheme is faster and the convergence performance is better than that of pc-efa algorithm.

\section{Conclusion}

Because the airborne phased array radar is flying at high altitude, it will receive a lot of clutter when it scans at high altitude. Therefore, clutter suppression becomes the main direction to improve the performance of phased array radar. 
At present, space-time adaptive processing technology (STAP) is the main technology to suppress clutter of phased array radar. However, STAP itself has many problems, so it can not be directly applied and so this paper proposes to optimize it. In this paper, EFA algorithm in STAP needs a large number of samples and computation, so a method of spatial data dimensionality reduction based on cyclic iterative calculation is adopted to optimize EFA algorithm. Finally, the experimental results show that the STAP performance of the optimized STAP algorithm is greatly improved, especially in the case of a small number of samples; the STAP performance of the EFA algorithm optimized in this paper is basically close to the ideal STAP performance; for clutter suppression in sidelobe clutter area, the optimization scheme in this paper also shows more excellent STAP performance; and compared with other optimization schemes, the optimization scheme in this paper is better in STAP performance and convergence speed, which shows that the optimization scheme in this paper is successful. However, the optimization scheme in this paper has only carried out simulation experiments, there is lack of practical application proof, and it is need to be further verified.

\section{Data Availability}

The raw/processed data required to reproduce these findings cannot be shared at this time as the data also form part of an ongoing study.

\section{Conflicts of Interest}

The authors declare that they have no conflicts of interest.

\section{Acknowledgments}

This project was granted financial support from the Key Project of the Sichuan Provincial Department of Education under Grant no. 18ZA0034.

\section{References}

[1] P. Setlur and M. Rangaswamy, "Waveform design for radar STAP in signal dependent interference," IEEE Transactions on Signal Processing, vol. 64, no. 1, pp. 19-34, 2016.

[2] Y. Fan, Y. Liu, Y. Liu, J. An, and X. Bu, "Robust two-stage reduced-dimension STAP algorithm and its performance analysis," Journal of Systems Engineering and Electronics, vol. 27, no. 5, pp. 954-960, 2016.

[3] J. Xu, S. Zhu, and G. Liao, "Range ambiguous clutters suppression for airborne FDA-STAP radar," IEEE Journal of Selected Topics in Signal Processing, vol. 9, no. 8, p. 1, 2015.

[4] M. K. Mcdonald and D. Cerutti-Maori, "Coherent radar processing in sea clutter environments, part 1: modelling and partially adaptive STAP performance," IEEE Transactions on Aerospace and Electronic Systems, vol. 52, no. 4, pp. 1797$1817,2016$.

[5] J. Xu, G. Liao, L. Huang et al., "Robust adaptive beamforming for fast-moving target detection with FDA-STAP radar," IEEE Transactions on Signal Processing, vol. 65, no. 4, pp. 973-984, 2016.
[6] B. Kang, "Student research highlight estimation of structured covariance matrices for radar STAP," IEEE Aerospace and Electronic Systems Magazine, vol. 30, no. 2, pp. 24-25, 2015.

[7] G. Yiduo, G. Jian, H. Darong et al., "Unified theoretical frame of a joint transmitter-receiver reduced dimensional STAP method for an airborne MIMO radar," Journal of Radars, vol. 5, no. 5, pp. 517-525, 2016.

[8] X. Wang, E. Aboutanios, and M. G. Amin, "Reduced-rank STAP for slow-moving target detection by antenna-pulse selection," IEEE Signal Processing Letters, vol. 22, no. 8, pp. 1150-1160, 2015.

[9] Y. Zhou, Z. Chen, L. R. Zhang et al., "Radar parameters aided STAP for heterogeneous clutter suppression," Journal of the University of Electronic Science and Technology of China, vol. 45, no. 6, pp. 905-910, 2016.

[10] H. Lin, Z. Yong-Shun, L. I. Zhe et al., "A dimension-reduced rapid STAP algorithm for airborne MIMO radar," Electronics Optics \& Control, vol. 7, no. 22, pp. 14-16, 2015.

[11] C. Shi-Wa, Z. Qing-Song, Z. Jian-Yun et al., "Signal characteristic analysis of scattered-wave fake clutter jamming method for STAP radar," Acta Electronica Sinica, vol. 46, pp. 401-409, 2018.

[12] Z. Gao and H. Tao, "Robust STAP algorithm based on knowledge-aided sparse recovery for airborne radar," Iet Radar Sonar \& Navigation, vol. 11, no. 2, pp. 321-329, 2016.

[13] B. L. McKinley, L. Kristine, and Bell, "Range-dependence compensation for bistatic STAP using focusing matrices," in Proceedings of the IEEE National Radar Conference-Proceedings, pp. 1750-1755, Arlington, VA, USA, May 2015.

[14] C. Wen, Y. J. Peng, and J. Wu, "Enhanced three-dimensional joint domain localized STAP for airborne FDA-MIMO radar under dense false-target jamming scenario," IEEE Sensors Journal, vol. 18, no. 10, pp. 4154-4166, 2018.

[15] S. Audrey, W. L. Melvin, and D. B. Williams, "Linear prediction based temporal weighting for pre-Doppler STAP," in Proceedings of the IEEE National Radar Conference, pp. 478-483, Arlington, VA, USA, May 2015.

[16] Z. Li, Y. Zhang, W. Liu et al., "Robust STAP against unknown mutual coupling based on middle subarray clutter covariance matrix reconstruction," IEEE Access, vol. 7, p. 1, 2019.

[17] Z. Chen, Y. Zhou, L. Zhang et al., "Single data set-STAP algorithm in heterogeneous environment," Hangkong Xuebao/Acta Aeronautica et Astronautica Sinica, vol. 36, no. 12, pp. 3938-3946, 2015.

[18] K. Duan, H. Xu, H. Yuan et al., "Reduced-DOFs three-dimension STAP via subarray synthesis for non-sidelooking planar array airborne radar," IEEE Transactions on Aerospace and Electronic Systems, vol. 56, no. 4, p. 1, 2019.

[19] G. Zhi-Qi, T. Hai-Hong, and Z. Ji-Chao, "Robust STAP algorithm based on joint sparse recovery of clutter spectrum for airborne radar," Acta Electronica Sinica, vol. 44, no. 11, pp. 2796-2801, 2016.

[20] F. Jia, Y. He, and R. Li, "Online antenna-pulse selection for STAP by exploiting structured covariance matrix," IEICE Transactions on Fundamentals of Electronics, Communications and Computer Sciences, vol. E102.A, no. 1, pp. 296-299, 2019. 